\title{
Correlation between thyroid autoantibodies and cardiovascular disease in patients with stages 3-5 chronic kidney disease
}

\author{
Weicheng Xu ${ }^{1,2} \wedge$, Shiyi Liang ${ }^{1 \wedge}$, Yuxiang Huang ${ }^{3 \wedge}$, Shili Zhao $^{2}$, Yunfang Zhang ${ }^{2}$, Yongqiang Li $^{3 \wedge}$ \\ ${ }^{1}$ Department of Nephrology, Institute of Nephrology and Urology, The Third Affiliated Hospital of Southern Medical University, Guangzhou, \\ China; ${ }^{2}$ Department of Nephrology, Huadu District People's Hospital, Southern Medical University, Guangzhou, China; ${ }^{3}$ Department of General \\ Practice, The Third Affiliated Hospital of Southern Medical University, Guangzhou, China \\ Contributions: (I) Conception and design: Y Li; (II) Administrative support: Y Zhang; (III) Provision of study materials or patients: Y Huang; (IV) \\ Collection and assembly of data: S Liang; W Xu; (V) Data analysis and interpretation: W Xu; (VI) Manuscript writing: All authors; (VII) Final \\ approval of manuscript: All authors. \\ Correspondence to: Yongqiang Li. Department of General Practice, The Third Affiliated Hospital of Southern Medical University, Guangzhou, \\ China. Email: liyongqiang851@163.com; Yunfang Zhang. Huadu District People's Hospital, Southern Medical University, 48\# Xinhua Road, Huadu \\ District, Guangzhou 510800, China. Email: zhang_yunf@163.com.
}

Background: Chronic kidney disease (CKD) is associated with thyroid disease and cardiovascular disease (CVD). To date, little is known about the association of thyroid autoantibodies with renal function or cardiac function in patients with CKD.

Methods: This is a descriptive cross-sectional study. Patients diagnosed with stages 3-5 CKD from January 2015 to May 2019 at our department were recruited. Routine medical history, general clinical data, and laboratory test indexes were collected for all patients. Echocardiography was performed by a trained echocardiographer to measure $\mathrm{E}$ in early diastole and $\mathrm{A}, \mathrm{E} / \mathrm{A}$ ratio, $\mathrm{E}$ ' in early diastole, $\mathrm{A}$ ' in end-diastole, E/E' ratio, and E'/A' ratio.

Results: A total of 1,164 patients with stages 3-5 CKD were included. Thyrotropin receptor antibody (TRAb) was significantly positively correlated with $C$-reactive protein $(r=0.206, P<0.001)$. Thyroid peroxidase antibody (TPOAb) and TGAb titers in male diabetic patients were higher $(\mathrm{r}=0.137, \mathrm{P}=0.023$; $\mathrm{r}=0.159, \mathrm{P}=0.011)$. In female patients, both TPOAb and TGAb were significantly negatively correlated with hemoglobin ( $\mathrm{r}=-0.213, \mathrm{P}=0.018 ; \mathrm{r}=-0.188, \mathrm{P}=0.019)$. The $\mathrm{E} / \mathrm{E}$ ' of patients who were TPOAb positive was higher than that in patients with TPOAb negative $(\mathrm{r}=0.181, \mathrm{P}<0.001)$. LVEF in patients who were TPOAb positive was higher than that in patients with TPOAb negative ( $\mathrm{r}=0.159, \mathrm{P}=0.007)$. In addition, LVEF was significantly negatively correlated with TRAb ( $\mathrm{r}=-0.112, \mathrm{P}=0.026)$.

Conclusions: In patients with stages 3-5 CKD, AITD may increase the risk of CVD in CKD patients by affecting triglycerides levels, increasing the risk of anemia, and promoting micro-inflammation. Attention should be paid to female patients with high TPOAb and TGAb titers. The mean of E/E' in patients with stage 5 CKD was 16.89 in the present study. Women with TPOAb positive may be more likely to develop diastolic heart failure.

Keywords: Chronic kidney disease (CKD); thyroid autoantibodies; cardiovascular disease (CVD); echocardiography

Submitted May 07, 2021. Accepted for publication Aug 11, 2021.

doi: $10.21037 /$ atm-21-3280

View this article at: https://dx.doi.org/10.21037/atm-21-3280

^ ORCID: Weicheng Xu, 0000-0003-1621-8787; Shiyi Liang, 0000-0002-9633-1642; Yuxiang Huang, 0000-0003-1329-8814; Yongqiang Li, 0000-0002-7572-2353. 


\section{Introduction}

Chronic kidney disease (CKD) has been recognized as a growing public health problem and affects $10-15 \%$ of the population worldwide (1). It is well established that cardiovascular disease (CVD) is the leading cause of morbidity and mortality in all patients with CKD, accounting for nearly half of all deaths $(2,3)$. Targeting modifiable factors has been frequently recommended as a first-line strategy for reducing kidney disease progression and CVD in patients with CKD. Although some studies have suggested an increased risk of coronary heart disease in autoimmune thyroiditis, the presence of thyroid autoantibodies does not appear to be associated with CVD risk in patients with subclinical hypothyroidism $(4,5)$. To date, little is known about the association of thyroid autoantibodies with renal function or cardiac function in patients with CKD. The aim of the present study was to investigate the relationship between thyroid autoimmunity and cardiac function of patients with stages 3-5 CKD. We present the following article in accordance with the STROBE reporting checklist (available at https://dx.doi. org/10.21037/atm-21-3280).

\section{Methods}

\section{Study population}

This is a descriptive cross-sectional study. We recruited a total of 1,477 patients ( $\geq 18$ years of age) with stages $3-5$ CKD, who attended the Department of Nephrology at the Third Affiliated Hospital of Southern Medical University from January 2015 to May 2019. Exclusion criteria were as follows: (I) patients with overt hypothyroidism [thyroidstimulating hormone $(\mathrm{TSH})>4.20 \mathrm{uIU} / \mathrm{L}]$ or overt hyperthyroidism (TSH $<0.27 \mathrm{uIU} / \mathrm{L}$ ); (II) patients lacking thyroid peroxidase antibody (TPOAb) and echocardiography data; and (III) patients whose thyroid function was altered due to previous use of thyroid medications. Finally, 1,164 patients (598 men and 566 women, mean age of $64.95 \pm 14.84$ years) were enrolled in the present study. The study was approved by the Ethics Committee of the Third Affiliated Hospital of Southern Medical University and was conducted according to the Declaration of Helsinki (as revised in 2013). All participants provided written informed consent.

\section{Clinical and laboratory examinations}

Fasting (>8 hours) blood samples were obtained to measure biochemical parameters. Hemoglobin (HGB), C-reactive protein (CRP), serum creatinine (SCr), blood urea nitrogen (BUN), uric acid, serum albumin, total cholesterol (TC), high-density lipoprotein cholesterol (HDL-C), lowdensity lipoprotein cholesterol (LDL-C), triglycerides (TG), creatine kinase (CK), CK isoenzyme (CK-MB), $\alpha$-hydroxybutyrate dehydrogenase (HBDH), troponin $\mathrm{T}$ (cTnT), myoglobin (Mb), n-terminal b-type natriuresis Peptide precursor (NT-proBNP), thyroid-stimulating hormone (TSH), TPOAb, thyroglobulin antibody (TGAb), and thyrotropin receptor antibody (TRAb) levels were measured. Roche cobas 6000 and Roche's reagents (Roche, Basel, Switzerland) were used to measure blood glucose, HDL-C, TG, and SCr by colorimetry, and BUN was measured by the rate method; LDL-C was calculated indirectly by HDL-C. Roche cobas 6000 and Orion's reagents (Orion, Espoo, Finland) were used to determine CRP by immune transmission nephelometry. Serum TSH levels (0.27-4.20 mIU/L), TGAb (0-115 IU/mL), TPOAb $(0-34 \mathrm{IU} / \mathrm{mL})$, and TRAb $(<1.58 \mathrm{U} / \mathrm{L})$ were measured by Beckman's automatic chemiluminescence immunoassay analyzer and Beckman Chemiluminescence Kit(Beckman, California, USA).

The estimated glomerular filtration rate (eGFR) was calculated using the modification of diet in renal disease equation (6):

$$
\begin{aligned}
& \operatorname{eGFR}\left(\mathrm{mL} \cdot \mathrm{min}^{-1} \cdot\left[\left[1.73 \mathrm{~m}^{2}\right]^{-1}\right]\right) \\
= & 175^{*} \mathrm{SCr}^{-1.234} \times \operatorname{age}^{-0.179} \times(0.79, \text { if female })
\end{aligned}
$$

Non-thyroidal illness syndrome is defined as patients with normal serum TSH. Autoimmune thyroid diseases (AITD) can be diagnosed when a patient has 1 or more positive thyroid autoantibody/autoantibodies and imaging evidence of abnormal thyroid function and/or imaging evidence of thyroid injury.

\section{Echocardiographic measurements}

All patients underwent 2-dimensional (DE), M-mode, pulsed, and color-flow Doppler echocardiographic examinations (EPIQ 7; Philips, Amsterdam, the Netherlands) in a left lateral position. Echocardiography was performed by a trained cardiac sonographer, who was blinded to the clinical status of the participants. Standard 2DE values for all 2DE parameters were obtained as average values of three consecutive cardiac 
cycles. Echocardiographic measurements were carried out following the Recommendations on Quantitative Methods of Adult Echocardiography Heart Cavity, published by the American Society of Echocardiography (ASE) in 2015 (7). The following measurements regarding diastolic dysfunction were assessed in all patients and controls: early diastolic trans-mitral flow velocity (E), late diastolic transmitral flow velocity (A), E/A ratio, early diastolic mitral annular velocity (E'), late diastolic mitral annular velocity (A'), E/E' ratio, and E'/A' ratio. Left ventricular ejection fraction (LVEF) was estimated by using the biplane method. Left ventricular diastolic function was measured based on the 2016 ASE/The European Association of Cardiovascular Imaging (EACVI) Recommendations on Echocardiographic Assessment of Left Ventricular Diastolic Function (8).

\section{Statistical analyses}

All statistical analyses were carried out using SPSS version 20.0 for Windows (Chicago, IL, USA). Variables with a normal distribution were expressed as the mean \pm standard deviation, and those with an abnormal distribution were expressed as the median (interquartile range). Categorical variables were expressed as proportions. Differences in clinical and laboratory values between patients with stages 3-5 CKD were assessed by Pearson's $\chi^{2}$-test, Wilcoxon test, or analysis of variance. Significant factors from the univariate analysis were included in multiple linear regression analyses to assess the association between thyroid status and echocardiographic parameters. Statistical correlations between factors in each group were analyzed using Pearson's correlation analysis. Our missing data analysis procedures used missing at random (MAR) assumptions. We used the MICE (multivariate imputation by chained equations) method of multiple multivariate imputation in SPSS. The statistical tests were 2 sided, and $\mathrm{P}<0.05$ was considered statistically significant.

\section{Results}

\section{Clinical and biochemical characteristics of the study participants}

A total of 1,882 patients with stages 3-5 CKD were screened. Of those, 16 patients younger than 18 years old, 134 patients with overt hypothyroidism, 68 patients with overt hyperthyroidism, 158 lacking TPOAb, 215 lacking echocardiography data, and 127 patients whose thyroid function was altered due to previous use of thyroid medications or drugs. Finally, 1,164 patients with stages 3-5 CKD were enrolled (566 women and 598 men). There were 340 patients with stage 3 CKD, 366 with stage 4 $\mathrm{CKD}$, and 458 with stage $5 \mathrm{CKD}$. Baseline data are shown in Table 1. The mean age of all stages was $>60$ years, of which the mean age for stage 3 was 65.06 years, the mean age for stage 4 was 66.47 years, and the mean age for stage 5 was 63.45 years. There was no statistically significant difference in age among patients with different stages $(\mathrm{P}=0.137)$. There was no statistically significant difference in the sex distribution among patients with different stages $(\mathrm{P}=0.909)$. The prevalence of hypertension in patients with stage 5 CKD was significantly higher than that in patients with stage 3 or $4(\mathrm{P}<0.001)$, while the prevalence of diabetes was lower in patients with stage $5 \mathrm{CKD}$ compared with patients with stage 3 or $4(\mathrm{P}=0.007)$. All patients with stages 3-5 CKD had anemia. Anemia becomes more severe with the loss of kidney function $(\mathrm{P}<0.001)$. Patients at all stages had hypertriglyceridemia, and there were statistically significant differences at each stage. The prevalence of AITD was statistically different among all stages $(\mathrm{P}=0.001)$. The prevalence of AITD in stages 3-5 CKD was $11.8 \%$, $14.2 \%$, and $21.4 \%$, respectively. Interestingly, there was no statistically significant difference in thyroid autoantibody titers among patients at different stages. However, the TPOAb positivity in patients with stage 5 CKD was significantly higher than that in stage 3 or $4 \mathrm{CKD}(\mathrm{P}=0.004)$. There were statistically significant differences in CRP and NT-proBNP among patients at different stages $(\mathrm{P}<0.001$ and $\mathrm{P}=0.021$, respectively), and both gradually increased with the deterioration of renal function. There was no significant difference in c $\mathrm{Tn} T$ between patients at different stages $(\mathrm{P}>0.05)$. For echocardiography parameters, there was no significant difference in E/A among patients at different stages $(\mathrm{P}=0.087)$, but multiple comparisons showed that the E/A of patients with stage $5 \mathrm{CKD}$ was higher than that in stage 4 CKD $(\mathrm{P}<0.05)$. There were statistically significant differences in $\mathrm{E} / \mathrm{E}$ ' among patients at different stages $(\mathrm{P}<0.001)$. However, multiple comparisons only showed that the E/E' of patients with stage $5 \mathrm{CKD}$ was higher than that in stages 3 and $4 \mathrm{CKD}$, while there was no significant difference between stages 3 and 4 CKD. To some extent, E'/A' is statistically different at each stage $(\mathrm{P}=0.053)$, although multiple comparisons only showed a significant difference in E'/A' between stages 4 and 5 CKD $(\mathrm{P}<0.05)$. The average LVEF of the included patients was $>50 \%$, and no significant systolic dysfunction was found. 
Table 1 Participants' characteristics according to stage $\left(\bar{x}_{ \pm s}\right)$

\begin{tabular}{|c|c|c|c|c|c|}
\hline Characteristics & Total $(n=1,164)$ & Stage $3(n=340)$ & Stage $4(n=366)$ & Stage $5(n=458)$ & $P$ value \\
\hline Age (years) & $64.95 \pm 14.84$ & $65.96 \pm 16.86$ & $66.47 \pm 14.41$ & $63.45 \pm 13.51$ & 0.137 \\
\hline Men, n (\%) & $598(51.4)$ & $178(52.4)$ & $186(50.8)$ & $234(51.1)$ & 0.909 \\
\hline Hypertension, n (\%) & $652(56.0)$ & $174(51.2)$ & $183(50.0)$ & $295(64.4)^{\star \#}$ & $<0.001$ \\
\hline HGB (g/L) & $96.29 \pm 24.74$ & $112.84 \pm 25.09$ & $91.50 \pm 17.96^{\star}$ & $88.16 \pm 22.51^{*}$ & $<0.001$ \\
\hline eGFR (mL/min/1.73 m²) & $21.46 \pm 16.43$ & $43.2 \pm 8.39$ & $21.62 \pm 4.24^{*}$ & $6.74 \pm 2.94^{\star \#}$ & $<0.001$ \\
\hline $\mathrm{SCr}(\mu \mathrm{mol} / \mathrm{L})$ & $471.16 \pm 384.14$ & $143.68 \pm 28.19$ & $261.16 \pm 58.46^{\star}$ & $808.7 \pm 340.43^{\star \#}$ & $<0.001$ \\
\hline BUN (mmol/L) & $16.85 \pm 9.71$ & $9.71 \pm 5.39$ & $15.01 \pm 6.68^{\star}$ & $22.69 \pm 9.79^{\star \#}$ & $<0.001$ \\
\hline $\mathrm{UA}(\mu \mathrm{mol} / \mathrm{L})$ & $432.85 \pm 142.52$ & $425.16 \pm 152.76$ & $441.5 \pm 141.87$ & $433.01 \pm 136.50$ & 0.672 \\
\hline HDL-C (mmol/L) & $1.15 \pm 0.32$ & $1.17 \pm 0.33$ & $1.17 \pm 0.3$ & $1.13 \pm 0.32$ & 0.485 \\
\hline LDL-C (mmol/L) & $1.69 \pm 1.11$ & $1.88 \pm 1.14$ & $1.62 \pm 1.15$ & $1.61 \pm 1.06^{*}$ & 0.065 \\
\hline VLDL-C (mmol/L) & $0.77 \pm 0.45$ & $0.79 \pm 0.47$ & $0.74 \pm 0.47$ & $0.78 \pm 0.43$ & 0.741 \\
\hline CK (IU/L) & $155.35 \pm 364.39$ & $145.38 \pm 498.88$ & $125.31 \pm 138.6$ & $176.55 \pm 355.99$ & 0.467 \\
\hline CK-MB (IU/L) & $18.40 \pm 22.41$ & $20.28 \pm 39.96$ & $16.89 \pm 6.96$ & $18.13 \pm 11.19$ & 0.511 \\
\hline HBDH (IU/L) & $181.30 \pm 83.70$ & $169.52 \pm 111.19$ & $187.83 \pm 77.22$ & $184.48 \pm 67.26$ & 0.196 \\
\hline cTnT (ng/mL) & $0.09 \pm 0.18$ & $0.07 \pm 0.22$ & $0.09 \pm 0.1$ & $0.11 \pm 0.17$ & 0.283 \\
\hline $\mathrm{Mb}(\mathrm{ng} / \mathrm{mL})$ & $193.76 \pm 206.74$ & $146.65 \pm 283.91$ & $167.56 \pm 167.61$ & $234.12 \pm 162.44^{\star \#}$ & $<0.001$ \\
\hline TPOAb positivity, n (\%) & $156(13.4)$ & $32(9.4)$ & $40(10.9)$ & $77(16.8)^{\star \#}$ & 0.004 \\
\hline TGAb positivity, n (\%) & $83(7.1)$ & $17(5.0)$ & $29(7.9)$ & $37(8.1)$ & 0.192 \\
\hline AITD, n (\%) & $190(16.3)$ & $40(11.8)$ & $52(14.2)$ & $98(21.4)^{\star \#}$ & 0.001 \\
\hline$E / A$ & $0.99 \pm 0.55$ & $0.98 \pm 0.56$ & $0.9 \pm 0.29$ & $1.05 \pm 0.63^{\#}$ & 0.087 \\
\hline E/E' & $11.46 \pm 10.24$ & $5.58 \pm 7.65$ & $7.61 \pm 7.57$ & $16.89 \pm 10.39^{\star \#}$ & $<0.001$ \\
\hline$E^{\prime} / A^{\prime}$ & $0.68 \pm 0.34$ & $0.68 \pm 0.24$ & $0.61 \pm 0.22$ & $0.71 \pm 0.43^{\#}$ & 0.053 \\
\hline LVEF\% & $65.32 \pm 8.21$ & $67.38 \pm 7.03$ & $65.42 \pm 6.83$ & $64.03 \pm 9.27^{\star}$ & 0.001 \\
\hline
\end{tabular}

*, significantly different $(P<0.05)$ from patients with stage 3 chronic kidney disease $(C K D)$; ", significantly different $(P<0.05)$ from patients with stage 4 CKD. AITD, autoimmune thyroid diseases; BUN, blood urea nitrogen; CK, creatine kinase; CK-MB: creatine kinase isoenzymes; CRP, C-reactive protein; CTnT, cardiac troponin T; E/A, early diastolic trans-mitral flow velocity/late diastolic transmitral flow velocity; E/E', early diastolic trans-mitral flow velocity/early diastolic mitral annular velocity; E'/A', early diastolic mitral annular velocity/late diastolic mitral annular velocity; eGFR, estimated glomerular filtration rate; $\mathrm{HBDH}$, hydroxybutyrate dehydrogenase; HDL-C, high density leptin cholesterol; HGB, hemoglobin; LDL-C, low-density lipoprotein cholesterol; LVEF, left ventricular ejection fraction; Mb, myoglobin; NT-proBNP, n-terminal b-type natriuretic peptide precursor; SCr, serum creatinine; TC, total cholesterol; TG, thyroglobulin; TGAb, thyroglobulin antibody; TPOAb, thyroid peroxidase antibody; TRAb, thyroid-stimulating hormone receptor antibody; TSH, thyroid-stimulating hormone; UA, uric acid; VLDL-C, very low-density lipoprotein cholesterol. 
Table 2 Relationship between renal function and thyroid autoantibody levels

\begin{tabular}{llll}
\hline Renal function & TPOAb & TGAb & TRAb \\
\hline SCr & $0.259^{*}$ & 0.259 & -0.011 \\
BUN & $0.311^{*}$ & 0.258 & -0.118 \\
eGFR & $-0.289^{*}$ & $-0.287^{*}$ & 0.202 \\
\hline
\end{tabular}

*, significant at $\mathrm{P}<0.05$ level. BUN, blood urea nitrogen; eGFR, estimated glomerular filtration rate; SCr, serum creatinine; TGAb, thyroglobulin antibody; TPOAb, thyroid peroxidase antibody; TRAb, thyroid-stimulating hormone receptor antibody.

Table 3 Multiple linear regression of thyroid autoantibodies and CVD risk factors

\begin{tabular}{|c|c|c|c|c|c|c|}
\hline Risk factors & $r$ & $P$ value & $r$ & $P$ value & $r$ & $P$ value \\
\hline Diabetes & 0.045 & 0.341 & 0.038 & 0.437 & 0.001 & 0.986 \\
\hline HGB & -0.073 & 0.127 & -0.075 & 0.126 & 0.014 & 0.772 \\
\hline TG & 0.012 & 0.803 & 0.046 & 0.347 & -0.078 & 0.110 \\
\hline TC & 0.043 & 0.370 & 0.041 & 0.403 & 0.038 & 0.436 \\
\hline HDL-C & 0.059 & 0.226 & 0.005 & 0.922 & 0.042 & 0.392 \\
\hline LDL-C & 0.016 & 0.735 & -0.031 & 0.531 & -0.002 & 0.973 \\
\hline
\end{tabular}

CRP, C-reactive protein; HDL-C, high density leptin cholesterol; HGB, hemoglobin; LDL-C, low-density lipoprotein cholesterol; TC, total cholesterol; TG, thyroglobulin; TGAb, thyroglobulin antibody; TPOAb, thyroid peroxidase antibody; TRAb, thyroid-stimulating hormone receptor antibody.

However, as the disease progresses, LVEF decreases. Multiple comparisons indicated that LVEF was slightly lower in patients with stage $5 \mathrm{CKD}$ than in patients with stage 3 CKD $(\mathrm{P}<0.05)$.

\section{Correlation between renal function and thyroid autoantibodies}

As shown in Table 2, the TPOAb titer is positively correlated with $\mathrm{SCr}$ and BUN $(\mathrm{r}=0.259, \mathrm{r}=0.311, \mathrm{P}<0.05)$ and negatively correlated with eGFR $(\mathrm{r}=-0.289, \mathrm{P}<0.05)$. There was a negative correlation between TGAb and eGFR ( $r=-0.287$, $\mathrm{P}<0.05)$, but there was no significant correlation between TGAb and SCr and BUN (P>0.05), whereas TRAb was not significantly associated with SCr, BUN, and eGFR $(\mathrm{P}>0.05)$.

\section{Correlation of thyroid autoantibodies with CVD risk factors}

As shown in Table 3, we used multiple linear regression to analyze the correlation between CVD risk factors and thyroid autoantibodies. After adjusting for age and sex, TPOAb and TGAb were found to be not significantly related to HGB, CRP, TG, TC, HDL-C, and LDL-C. A significant positive correlation was observed between $\mathrm{TRAb}$ and CRP $(\mathrm{r}=0.206, \mathrm{P}<0.001)$.

As shown in Table 4, TPOAb and TGAb titers and positivity were statistically different between male and female patients. The correlations between diabetes, hypertension, HGB, CRP, TG, TC, HDL-C, and LDL-C levels and TPOAb and TGAb were statistically analyzed between different sexes.

As shown in Tables 5 and 6, men with diabetes had higher TPOAb and TGAb titers $(r=0.137, \mathrm{P}=0.023 ; \mathrm{r}=0.159$, $\mathrm{P}=0.011$ ), whereas there was no significant correlation between TPOAb and TGAb with hypertension, HGB, CRP, TG, TC, HDL-C, and LDL-C ( $>0.05)$.

In female patients, TPOAb and TGAb were significantly negatively correlated with HGB $(\mathrm{r}=-0.213, \mathrm{P}=0.018$; $\mathrm{r}=-0.188, \mathrm{P}=0.019)$. There was no significant correlation 
Table 4 Sex differences in thyroid autoantibodies $(\bar{x} \pm \mathrm{s})$

\begin{tabular}{lccc}
\hline Autoantibodies & Men & Women & P value \\
\hline TPOAb & $19.45 \pm 40.31$ & $38.64 \pm 87.98$ & 0.001 \\
TGAb & $68.57 \pm 341.44$ & $256.06 \pm 815.96$ & 0.002 \\
TRAb & $0.40 \pm 0.16$ & $0.40 \pm 0.18$ & 0.780 \\
TPOAb positivity, $\mathrm{n}(\%)$ & $58(9.1)$ & $102(19.4)$ & $<0.001$ \\
TGAb positivity, $\mathrm{n}(\%)$ & $17(2.7)$ & $78(14.8)$ & $<0.001$ \\
\hline
\end{tabular}

TGAb, thyroglobulin antibody; TPOAb, thyroid peroxidase antibody; TRAb, thyroid-stimulating hormone receptor antibody.

Table 5 Multiple linear regression analysis of TPOAb and risk factors for CVD among sexes

\begin{tabular}{lcccc}
\hline \multirow{2}{*}{ Risk factors } & \multicolumn{2}{c}{ Men } & & Women \\
\cline { 2 - 5 } Hypertension & $r$ & P value & r & 0.983 \\
Diabetes & -0.018 & 0.656 & 0.002 & 0.707 \\
HGB & 0.137 & 0.023 & -0.029 & 0.018 \\
CRP & 0.041 & 0.495 & -0.213 & 0.043 \\
TG & -0.026 & 0.666 & 0.042 & 0.580 \\
TC & -0.021 & 0.729 & 0.112 & 0.155 \\
HDL-C & -0.012 & 0.843 & 0.166 & 0.034 \\
LDL-C & -0.048 & 0.423 & 0.037 & 0.642 \\
\hline
\end{tabular}

CRP, C-reactive protein; HDL-C, high density leptin cholesterol; HGB, hemoglobin; LDL-C, low-density lipoprotein cholesterol; TC, total cholesterol; TG, thyroglobulin.

Table 6 Multiple linear regression analysis of TGAb and risk factors for CVD among sexes

\begin{tabular}{lcccc}
\hline \multirow{2}{*}{ Risk factors } & \multicolumn{2}{c}{ Men } & & \multicolumn{2}{c}{ Women } \\
\cline { 2 - 5 } Hypertension & $r$ & P value & P value & 0.067 \\
Diabetes & 0.041 & 0.504 & -0.047 & 0.401 \\
HGB & 0.159 & 0.011 & -0.188 & 0.019 \\
CRP & 0.045 & 0.464 & -0.034 & 0.669 \\
TG & -0.040 & 0.518 & 0.102 & 0.200 \\
TC & -0.022 & 0.721 & 0.093 & 0.247 \\
HDL-C & 0.005 & 0.933 & 0.066 & 0.410 \\
LDL-C & -0.060 & 0.335 & -0.065 & 0.420 \\
\hline
\end{tabular}

CRP, C-reactive protein; HDL-C, high density leptin cholesterol; HGB, hemoglobin; LDL-C, low-density lipoprotein cholesterol; TC, total cholesterol; TG, thyroglobulin. 
Table 7 Multiple linear regression of thyroid autoantibodies and cardiac function markers

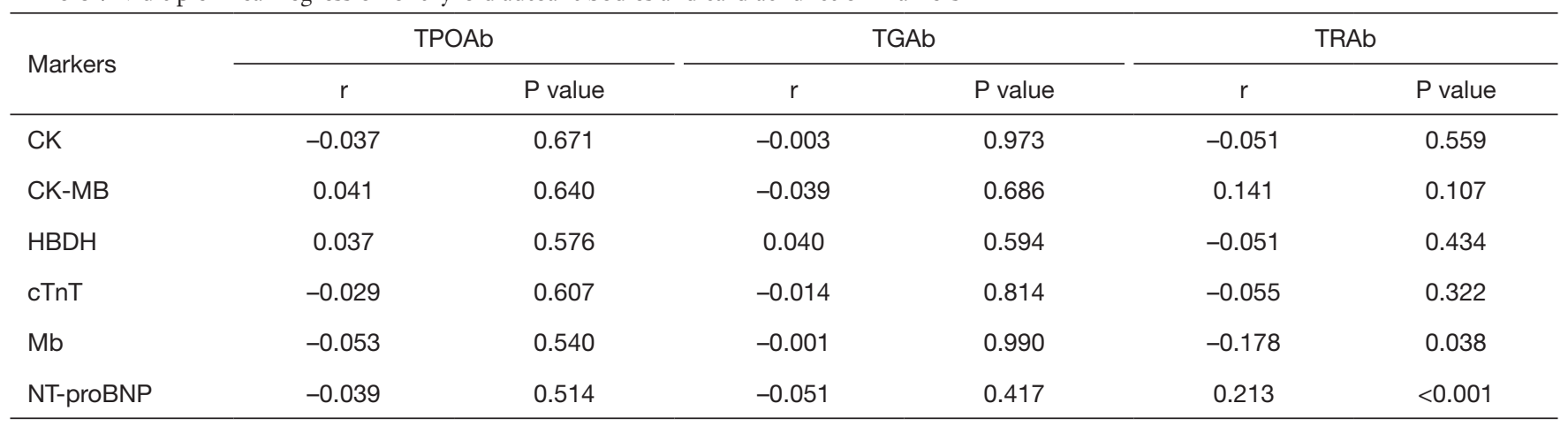

$\mathrm{CK}$, creatine kinase; CK-MB, creatine kinase isoenzymes; cTnT, cardiac troponin T; HBDH, hydroxybutyrate dehydrogenase; Mb, myoglobin; NT-proBNP, n-terminal b-type natriuretic peptide precursor.

Table 8 Multiple linear regression of TRAb and Mb and NT-proBNP in female patients

\begin{tabular}{lcc}
\hline \multirow{2}{*}{ Markers } & \multicolumn{2}{c}{ TRAb } \\
\cline { 2 - 3 } & $r$ & P value \\
\hline Mb & -0.190 & 0.015 \\
NT-proBNP & 0.313 & $<0.001$ \\
\hline
\end{tabular}

Mb, myoglobin; NT-proBNP, n-terminal b-type natriuretic peptide precursor.

between TPOAb and TGAb for hypertension, diabetes, CRP, TG, TC, HDL-C, and LDL-C $(\mathrm{P}>0.05)$.

\section{Correlation of thyroid autoantibodies with markers of cardiac function}

As shown in Table 7, after adjusting for age and sex, there was no significant correlation between TPOAb and TGAb and CK, CK-MB, HBDH, cTnT, Mb, and NT-proBNP. However, TRAb was significantly negatively correlated with $\mathrm{Mb}(\mathrm{r}=-0.178, \mathrm{P}=0.038)$, and positively correlated with NTproBNP $(r=0.213, \mathrm{P}<0.001)$. Breaking the data down by sex, we found that TRAb was only associated with $\mathrm{Mb}$ and NTproBNP in women $(\mathrm{r}=-0.190, \mathrm{P}=0.015 ; \mathrm{r}=0.313, \mathrm{P}<0.001)$. There was no significant correlation between TPOAb and TGAb with CK, CK-MB, HBDH, cTnT, Mb, and NTproBNP between men and women, as shown in Table 8 .

\section{Correlation of thyroid autoantibodies with echocardiographic parameters}

We used multiple linear regression to analyze the correlation between thyroid autoantibodies and E/A, E/ E', E'/A', and LVEF. After adjusting for age and sex, E/A was found to be not associated with thyroid autoantibodies $(\mathrm{P}>0.05)$. Patients who were TPOAb positive had higher $\mathrm{E} / \mathrm{E}$ ' $(\mathrm{r}=0.181, \mathrm{P}<0.001)$, while there was no significant correlation between E/E' and TPOAb, TGAb, TRAb, and TGAb positivity $(\mathrm{P}>0.05)$. Interestingly, patients who were TPOAb positive had higher LVEF ( $\mathrm{r}=0.159, \mathrm{P}=0.007)$. In addition, LVEF was significantly negatively correlated with TRAb ( $\mathrm{r}=-0.112, \mathrm{P}=0.026)$. There was no significant correlation between E'/A' and TPOAb, TGAb, TRAb, TPOAb and TGAb positivity $(\mathrm{P}>0.05)$, as shown in Table 9.

\section{Discussion}

The prevalence and mortality of CKD is increasing worldwide. Despite rising medical standards and an increase in the average survival time of patients, CKD will eventually lead to end-stage renal disease (ESRD), which poses a great challenge. There is a close association between the thyroid and kidneys. Thyroid autoantibodies and their antigens may be deposited in the glomeruli and cause kidney damage. In addition, immune abnormalities in AITD patients may lead to secondary renal disease. Compared with the normal population, the prevalence of thyroid dysfunction is higher in patients with CKD. Patients with CKD often have CVD in the early stage, and CVD is the leading cause of death in patients with CKD.

A total of 1,164 patients with stages 3-5 CKD were enrolled in our study (566 women and 598 men). A total of 340 patients had stage $3 \mathrm{CKD}, 366$ had stage $4 \mathrm{CKD}$, and 458 had stage 5 CKD. We found that the prevalence of AITD gradually increased with the deterioration of 
Table 9 Multiple linear regression analysis of thyroid autoantibodies and echocardiographic parameters

\begin{tabular}{|c|c|c|c|c|c|c|c|c|}
\hline Autoantibodies & \multicolumn{2}{|c|}{$E / A$} & \multicolumn{2}{|c|}{$E / E$} & \multicolumn{2}{|c|}{$E^{\prime} / A^{\prime}$} & \multicolumn{2}{|c|}{ LVEF } \\
\hline TPOAb & -0.029 & 0.563 & 0.106 & 0.105 & 0.006 & 0.900 & -0.054 & 0.407 \\
\hline TGAb & -0.045 & 0.369 & 0.099 & 0.129 & 0.008 & 0.869 & -0.069 & 0.291 \\
\hline TRAb & 0.068 & 0.175 & 0.029 & 0.561 & 0.031 & 0.539 & -0.112 & 0.026 \\
\hline TGAb positivity & -0.063 & 0.208 & -0.069 & 0.179 & -0.041 & 0.426 & -0.057 & 0.338 \\
\hline
\end{tabular}

E/A, early diastolic trans-mitral flow velocity/late diastolic transmitral flow velocity; E/E', early diastolic trans-mitral flow velocity/early diastolic mitral annular velocity; E'/A', early diastolic mitral annular velocity/late diastolic mitral annular velocity; LVEF, left ventricular ejection fraction; Mb, myoglobin; TGAb, thyroglobulin antibody; TPOAb, thyroid peroxidase antibody; TRAb, thyroid-stimulating hormone receptor antibody.

renal function, and NT-proBNP and CRP and TPOAb and TGAb titers gradually increased. The positive rate of TPOAb, E/A, E/E', and E'/A' were higher in patients with stage $5 \mathrm{CKD}$. The TG of patients at each stage was high, and the increase of TG in stage 5 CKD was more obvious. Studies have shown that CVD is closely related to changes in body composition, such as decreased muscle tissue and increased fat tissue (9-11). A study in China pointed out that the reduction of skeletal muscle index is independently related to the occurrence of CVD in CKD patient (12). Our study showed that triglyceride of patients at each stage was high, and the increase of TG in stage 5 CKD was more obvious. This means that with the progress of CKD, the disorder of blood lipid metabolism may become more obvious, and the risk of cardiovascular disease will also increase. The prevalence of AITD was statistically different at each stage, and the prevalence was $11.8 \%$ for stage 3 , $14.2 \%$ for stage 4 , and $21.4 \%$ for stage 5 CKD. In patients with stages 3-5 CKD, TRAb was significantly positively correlated with CRP and negatively correlated with $\mathrm{Mb}$. It was only positively correlated with NT-proBNP in female patients. There were significant differences between TPOAb and TGAb among sexes. Men with diabetes have higher levels of TPOAb and TGAb than their female counterparts. Among female patients, TPOAb and TGAb are significantly negatively correlated with HGB. TPOAbpositive patients had higher E/E' and LVEF, while patients with higher TRAb titers had lower LVEF.

The lack of this study is that the impact of arrhythmia is ignored due to the lack of ECG data, and the data is not stratified by the primary disease.

\section{Thyroid immune disorder and CKD}

Similar immune complex deposits were observed in the thyroid follicular epithelium and glomerular basement membrane in patients with glomerulonephritis associated with Hashimoto's thyroiditis. The same circulating immune complex can be involved in both diseases (13). Hasnain et al. found there was deposition of TPOAb in the renal tissues of patients with membranous nephropathy complicated with Graves' disease. And it suggested that TPOAb may contribute to the genesis of membranous nephropathy (14). Therefore, some autoimmune-mediated glomerulonephritis and AITD may have similar pathogeneses.

The findings of our study indicated that the prevalence of AITD gradually increased in patients with stages 3-5 CKD. TPOAb positivity in patients with stage $5 \mathrm{CKD}$ was significantly higher than that in patients with stages 3 and 4 CKD. With the decrease of eGFR, TPOAb and TGAb titers gradually increased, suggesting that there may be some interaction between AITD and CKD. There may be antigenic cross-reactions between AITD and CKD, but these hypotheses require further research to confirm. It has been reported that patients with hyperthyroidism develop membranous nephropathy after treatment with propylthiouracil, and iodine-131 treatment can lead to anti-neutrophil cytoplasmic antibodies (ANCA)-positive crescent nephritis $(15,16)$. The pathogenesis remains to be further investigated.

\section{Correlation between thyroid immune disorder and echocardiographic parameters}

Echocardiography has the advantages of accurate, objective, 
reproducible, and safe operation in evaluating cardiac function. It can display patients' ventricular systolic and diastolic processes, cardiac cavity structure, and blood flow. NT-proBNP may be used to diagnose and evaluate the prognosis of heart failure, but it is not used to distinguish the types of heart failure. Echocardiography cannot only be used to evaluate the cardiac function and prognosis of patients but can also be used to classify the types of heart failure. Studies have shown that the sensitivity and specificity of various echocardiographic parameters in evaluating ejection fraction-retaining heart failure are high, $95 \%$ and $100 \%$, respectively (17). Echocardiography plays an important role in ejection fraction-retaining heart failure. Echocardiography can evaluate the left ventricular diastolic function by measuring the E peak, A peak, E/A, E peak deceleration time, $\mathrm{A}^{\prime}$, and E/E'. Kasner et al. found that E/E' examined by tissue doppler imaging (TDI) and pulsed wave doppler $(\mathrm{PW})$ can better assess left ventricular diastolic function (18). Similarly, studies have shown that E/E' is significant in evaluating left ventricular filling pressure and can predict the occurrence of adverse events during ESRD $(19,20)$. De Sutter et al. noted that E'/A' decreases and E/ $\mathrm{E}$ ' increases with age (21). These results are consistent with the results of our study. A study showed the decreased diastolic function and cardiac structure damage are common in CKD3 patients (22). Heart damage is more severe in CKD3 patients with Yin-Yang deficiency syndrome than that in spleen-kidney Yang deficiency syndrome and QiYin deficiency syndrome, and the impairment of cardiac function is most severe in Yin-Yang deficiency syndrome. The complication of pathogen-excess can aggravate heart damage. Patients complicated with blood-stasis syndrome have more severe myocardial hypertrophy and ventricular and atrial enlargement degree.

The findings of the present study indicated that the mean $\mathrm{E} / \mathrm{E}$ ' in patients with stage $5 \mathrm{CKD}$ was 16.89 , which was higher than that in stages 3 and 4 . Patients with stage 5 CKD have already developed ejection fraction-preserving heart failure. The E/E' of patients who are TPOAb positive is higher, suggesting that patients who are TPOAb positive may be more prone to diastolic dysfunction, and early intervention should be provided. Only patients with stage $5 \mathrm{CKD}$ were found to have higher E'/A', but there was no statistically significant difference in the analysis of E'/A' in patients with stages 3-5 CKD. E'/A' was also not found to be significantly related to thyroid autoantibodies, which is consistent with guidelines that do not recommend the use of E'/A' to assess cardiac function (23). Patients who were
TPOAb positive had higher LVEF, while TPOAb had a negative correlation with HGB in female patients, and anemia may increase LVEF. The specific mechanism needs further study. Patients with higher TRAb had lower LVEF, and TRAb was significantly positively correlated with CRP. CRP can promote the formation of atherosclerosis can also cause coronary spasm, trigger myocardial ischemia, and reduce LVEF. For E/A, E/E', E'/A', and LVEF, no significant difference was found between stages 3 and 4 CKD. Cardiac dysfunction that can be detected by echocardiography may not appear until CKD progresses to stage 5 . Once CKD progresses to stage 5 , heart dysfunction may develop.

\section{Conclusions}

The prevalence of AITD in patients with stages 3-5 CKD was $11.8 \%, 14.2 \%$, and $21.4 \%$, respectively. With the decline of renal function, the prevalence of AITD increased gradually, and TPOAb and TGAb titers gradually increased. The average E/E' of patients with stage $5 \mathrm{CKD}$ was 16 . Women who are TPOAb positive may be more prone to diastolic heart failure. TRAb may reduce LVEF by promoting inflammation or triggering coronary spasm. Thyroid immune disorder in patients with CKD need more attention, and thyroid autoantibodies should be checked as a basic item.

\section{Acknowledgments}

Funding: The present study was supported by the Thirteenth Five-Year Plan of Guangdong Province Educational Science (No. 2020GXJK441), the Project of Traditional Chinese Medicine Bureau of Guangdong Province (No. 20191228 to ZY), Guangzhou Traditional Chinese Medicine and Integrated Chinese and Western Medicine Science and Technology Project (No. 20202A010023), and the Intramural Research Program of People's Hospital of Huadu District (No. 2020C03), and the Intramural Research Program of People's Hospital of Huadu District (No. 2019B01).

\section{Footnote}

Reporting Checklist: The authors have completed the STROBE reporting checklist. Available at https://dx.doi. org/10.21037/atm-21-3280

Data Sharing Statement: Available at https://dx.doi. 


\section{Page 10 of 11}

org/10.21037/atm-21-3280

Conflicts of Interest: All authors have completed the ICMJE uniform disclosure form (available at https://dx.doi. org/10.21037/atm-21-3280). Dr. WX reports funding support from the Intramural Research Program of People's Hospital of Huadu District (No. 2020C03). Dr. SZ reports funding support from the Guangzhou Traditional Chinese Medicine and Integrated Chinese and Western Medicine Science and Technology Project (No. 20202A010023), and the Intramural Research Program of People's Hospital of Huadu District (No. 2020C03). Dr. YL reports funding support from the Thirteenth Five-Year Plan of Guangdong Province Educational Science (No. 2020GXJK441). The other authors have no conflicts of interest to declare.

Ethical Statement: The authors are accountable for all aspects of the work in ensuring that questions related to the accuracy or integrity of any part of the work are appropriately investigated and resolved. The study was approved by the Ethics Committee of the Third Affiliated Hospital of Southern Medical University and was conducted according to the Declaration of Helsinki (as revised in 2013). All participants provided written informed consent.

Open Access Statement: This is an Open Access article distributed in accordance with the Creative Commons Attribution-NonCommercial-NoDerivs 4.0 International License (CC BY-NC-ND 4.0), which permits the noncommercial replication and distribution of the article with the strict proviso that no changes or edits are made and the original work is properly cited (including links to both the formal publication through the relevant DOI and the license). See: https://creativecommons.org/licenses/by-nc-nd/4.0/.

\section{References}

1. Hill NR, Fatoba ST, Oke JL, et al. Global Prevalence of Chronic Kidney Disease - A Systematic Review and MetaAnalysis. PLoS One 2016;11:e0158765.

2. Collins AJ, Foley RN, Gilbertson DT, et al. United States Renal Data System public health surveillance of chronic kidney disease and end-stage renal disease. Kidney Int Suppl (2011) 2015;5:2-7.

3. Wheeler D, Hayes R, Landray M, et al. Taal: Brenner and Rector's The Kidney. 9th edn Philadelphia, PA, USA: Elsevier Saunders, 2012:2060-75.

4. Chen WH, Chen YK, Lin CL, et al. Hashimoto's thyroiditis, risk of coronary heart disease, and L-thyroxine treatment: a nationwide cohort study. J Clin Endocrinol Metab 2015;100:109-14.

5. Wells BJ, Hueston WJ. Are thyroid peroxidase antibodies associated with cardiovascular disease risk in patients with subclinical hypothyroidism? Clin Endocrinol (Oxf) 2005;62:580-4.

6. Ma YC, Zuo L, Chen JH, et al. Modified glomerular filtration rate estimating equation for Chinese patients with chronic kidney disease. J Am Soc Nephrol 2006;17:2937-44.

7. Lang RM, Badano LP, Mor-Avi V, et al. Recommendations for cardiac chamber quantification by echocardiography in adults: an update from the American Society of Echocardiography and the European Association of Cardiovascular Imaging. Eur Heart J Cardiovasc Imaging 2015;16:233-70.

8. Baumgartner H, Hung J, Bermejo J, et al. Recommendations on the Echocardiographic Assessment of Aortic Valve Stenosis: A Focused Update from the European Association of Cardiovascular Imaging and the American Society of Echocardiography. J Am Soc Echocardiogr 2017;30:372-92.

9. Chen GC, Arthur R, Iyengar NM, et al. Association between regional body fat and cardiovascular disease risk among postmenopausal women with normal body mass index. Eur Heart J 2019;40:2849-55.

10. Arias Téllez MJ, Acosta FM, Sanchez-Delgado G, et al. Association of Neck Circumference with Anthropometric Indicators and Body Composition Measured by DXA in Young Spanish Adults. Nutrients 2020;12:514.

11. Yilmaz Z, İnce H, Aydin E, et al. Relationship Between Epicardial Adipose Tissue and Body Composition as Determined by Multi-Frequency Bioelectrical Impedance Analysis in Patients with Stage 5 Chronic Kidney Disease. Med Sci Monit 2020;26:e920233.

12. Zheng $\mathrm{K}$, Liu C, Lin C, et al. Related factors analysis of body composition and cardiovascular disease in patients with chronic kidney disease. Chinese Journal of Nephrology 2021;37:1-7.

13. Thajudeen B, John SG, Ossai NO, et al. Membranous nephropathy with crescents in a patient with Hashimoto's thyroiditis: a case report. Medicine (Baltimore) 2014;93:e63.

14. Hasnain W, Stillman IE, Bayliss GP. Minimal-change renal disease and Graves' disease: a case report and literature review. NDT Plus 2011;4:96-8.

15. Mariani LH, Berns JS. The renal manifestations of thyroid 
disease. J Am Soc Nephrol 2012;23:22-6.

16. Wang C, Gou SJ, Xu PC, et al. Epitope analysis of antimyeloperoxidase antibodies in propylthiouracil-induced antineutrophil cytoplasmic antibody-associated vasculitis. Arthritis Res Ther 2013;15:R196.

17. Arteaga RB, Hreybe H, Patel D, et al. Derivation and validation of a diagnostic model for the evaluation of left ventricular filling pressures and diastolic function using mitral annulus tissue Doppler imaging. Am Heart J 2008;155:924-9.

18. Kasner M, Westermann D, Steendijk P, et al. Utility of Doppler echocardiography and tissue Doppler imaging in the estimation of diastolic function in heart failure with normal ejection fraction: a comparative Doppler-conductance catheterization study. Circulation 2007;116:637-47.

19. Nagueh SF, Middleton KJ, Kopelen HA, et al. Doppler tissue imaging: a noninvasive technique for evaluation of left ventricular relaxation and estimation of filling pressures. J Am Coll Cardiol 1997;30:1527-33.

20. Sharma R, Pellerin D, Gaze DC, et al. Mitral peak

Cite this article as: $\mathrm{Xu} \mathrm{W}$, Liang S, Huang Y, Zhao S, Zhang Y, Li Y. Correlation between thyroid autoantibodies and cardiovascular disease in patients with stages 3-5 chronic kidney disease. Ann Transl Med 2021;9(16):1301. doi: 10.21037/atm$21-3280$
Doppler E-wave to peak mitral annulus velocity ratio is an accurate estimate of left ventricular filling pressure and predicts mortality in end-stage renal disease. J Am Soc Echocardiogr 2006;19:266-73.

21. De Sutter J, De Backer J, Van de Veire N, et al. Effects of age, gender, and left ventricular mass on septal mitral annulus velocity ( $\left.E^{\prime}\right)$ and the ratio of transmitral early peak velocity to E' (E/E'). Am J Cardiol 2005;95:1020-3.

22. Pan WL, Sun Y, Li XF. Relationship of Cardiac Damage with Traditional Chinese Medicine Syndromes and Clinical Indicators in Patients with Chronic Kidney Disease at Stage 3. Journal of Guangzhou University of Traditional Chinese Medicine 2018;35:197-205.

23. Nagueh SF, Smiseth OA, Appleton CP, et al. Recommendations for the Evaluation of Left Ventricular Diastolic Function by Echocardiography: An Update from the American Society of Echocardiography and the European Association of Cardiovascular Imaging. Eur Heart J Cardiovasc Imaging 2016;17:1321-60.

(English Language Editor: J. Jones) 